\title{
CRITICAL POINTS OF THE DISPLACEMENT FUNCTION OF AN ISOMETRY
}

\author{
VILNIS OZOLS
}

\section{Introduction}

Given a Riemannian manifold $M$ and a group of isometries of $M$ it is natural to study the fixed point set of this group. This problem was considered by $S$. Kobayashi in [9], [10], and by R. Bott in [2], in the case where the group is a 1-parameter group of isometries. In [4], Kobayrashi shows that if $\left\{g_{\imath}\right\}$ is such a group, then the fixed point set of $\left\{g_{t}\right\}$ is a totally geodesic submanifold of even codimension. In fact, his proof shows that the fixed point set of any group of isometries is a totally geodesic submanifold. The fixed point set of the 1-parameter group $\left\{g_{t}\right\}$ is just the set of zeros of the associated Killing vector field $X$, and in [7] and [8] R. Hermann considers the more general problem of the critical points of the function $|X|^{2}$ giving the square of the length of $X$. He shows that these critical points are exactly the points lying on geodesic orbits of $\left\{g_{t}\right\}$. Moreover, he shows that if $M$ has curvature $K \leq 0$, then the set of critical points of $\left|X^{2}\right|$ is convex (that is, any geodesic segment between two critical points lies in the critical set).

We consider the still more general situation of a single isometry $f$, and look at the critical point set Crit $(f)$ of the function $\delta_{f}^{2}$, where $\delta_{f}(x)=$ distance $(x, f(x))$. It is evident that Crit $(f)$ contains the fixed points of $f$.

In Chapter I we let $M$ be any Riemannian manifold and $f: M \rightarrow M$ an isometry whose displacement $\delta_{f}$ is small enough so that $f$ takes each point into the complement of its cut locus. We say such an isometry has "small displacement." The main theorems are:

(1.2.1) Theorem. Let $f: M \rightarrow M$ be an isometry of small displacement and $x \in M$. Then $x \in \mathrm{Crit}(f)$ if and only if $f$ preserves the unique minimizing geodesic between $x$ and $f(x)$.

(1.3.4) Theorem. Let $M$ have curvature $K \leq 0$, and assume $f: M \rightarrow M$ is an isometry of small displacement: Then

(i) Crit $(f)$ is a totally geodesic submanifold possibly with boundary,

(ii) $\delta_{f}$ takes its absolute minimum on Crit $(f)$.

Received June 18, 1968. The author wishes to thank Professor J. A. Wolf for his guidance and much helpful discussion in the preparation of this work, and would also like to thank Professor S. Kobayashi for his valuable suggestions. 
(iii) If Fix $(f)=\emptyset$ then Crit $(f)$ is connected; if Fix $(f) \neq \emptyset$ then Crit $(f)$ $=$ Fix $(f)$.

(iv) If $M$ is simply connected then Fix $(f)$ is connected.

(v) If $K<0$ and Fix $(f)=\emptyset$, then Crit $(f)$ is either empty or consists of a single geodesic.

Moreover, we show that if $f \in I^{0}(M)=$ identity component of the isometry group of $M$, and $f=f_{1}$, where $\left\{f_{t}\right\}$ is a 1-parameter group of isometries with associated Killing vector field $X$, then Crit $\left(|X|^{2}\right)=\bigcap_{n=1}^{\infty}$ Crit $\left(f_{1 / n}\right)$, so that our results in a sense generalize those of $\mathbf{R}$. Hermann in [8].

In Chapter II we restrict to Riemannian homogeneous spaces and principally to symmetric spaces. The main theorem is:

(2.7.1) Theorem. Let $M$ be a simply connected Riemannian symmetric space with $M=M_{0} \times M_{1} \times \cdots M_{k}$, where $M_{0}$ is a Euclidean space and the $M_{i}, 1 \leq i \leq k$, are irreducible. If $g \in I^{0}(M)=I^{\circ}\left(M_{0}\right) \times \cdots \times I^{\circ}\left(M_{k}\right)$, and the components $g_{i}$ of $g$ which act on the compact $M_{i}$ are sufficiently close to the identity, then the components of Crit $(g)$ are the orbits $Z_{I 0(M)}^{0}(g) \cdot x$, where $x$ is any point in the component, and $Z_{I^{\circ}(M)}^{0}(g)$ is the identity component of the centralizer of $g$ in $I^{\circ}(M)$. (Here $I^{\circ}(M)=$ identity component of the isometry group of $M$ ).

If the isometry $g$ is sufficiently near the identity, it lies on a unique 1-parameter group $\left\{g_{t}\right\}$ of isometries, with associated Killing vector field $X$. If $M$ is symmetric, we show that Crit $\left(|X|^{2}\right)=\operatorname{Crit}\left(g_{t}\right)$ for any $t \in(0,1]$. We then obtain an explicit formula for the Hessian of the function $|X|^{2}$, and show that Crit $\left(|X|^{2}\right)$ is a non-degenerate critical sub-manifold in the sense of $\mathrm{R}$. Bott [1] if $M$ is either of non-compact type, or if $M$ is of compact type and $X$ is a regular element of the Lie algebra of the isometry group.

Notation. We adopt the notation used in the book of Kobayashi-Nomizu [11] for Riemannian manifolds, and refer to the books of S. Helgason [6] and J. A. Wolf [15] for the basic facts about symmetric spaces and Lie groups. In a homogeneous space $M=G / K$ we assume we have a fixed direct sum decomposition $\boldsymbol{G}=\boldsymbol{K}+\boldsymbol{m}$, where $\boldsymbol{G}$ is the Lie algebra of $\boldsymbol{G}, \boldsymbol{K}$ the Lie algebra of $K$, and $m$ a complementary subspace satisfying ad $(K) m \subset m$. This is a reductive homogeneous space. We assume $M$ has an invariant Riemannian metric $B^{*}$, and let $B$ be its restriction to $m \times m$, where $m$ is naturally identified with the tangent space of $M$ at $K$. Then we say $B^{*}$ is a normal metric if $B\left([X, Z]_{m}, Y\right)+B\left(X,[Y, Z]_{m}\right)=0$ for $X, Y, Z \in m$. A normal metric induces a Riemannian connection of type (A1) in the notation of Nomizu [12], and this connection is characterized by the fact that its geodesics are the translates $g x(s)$, where $x(s)=(\exp s T) \cdot T$ and $g \in G, T \in m$. 


\section{Chapter I. The general case}

(1.1) $M$ will always be a complete connected Riemannian manifold with metric $g$ and Riemannian connection $\nabla$. Let $\rho$ be the distance on $M$ induced by $g$ and defined by: $\rho(x, y)=\inf _{p}$ \{length $p \mid p$ is a piecewise smooth path from $x$ to $y$ \}. "Smooth" and "differentiable" will always mean $C^{\infty}$, and $T(M)$ denotes the tangent bundle of $M$. Because of completeness, exp: $T(M) \rightarrow M$ is defined, surjective, and smooth. If $f: M \rightarrow N$ is a smooth map, $f_{*}: T(M) \rightarrow T(N)$ is the induced map on the tangent bundles. For every smooth map $f: M \rightarrow M$ we define the displacement function $\delta_{f}: M \rightarrow R$ (= real numbers) by $\delta_{f}(x)=$ $\rho(x, f(x))$.

(1.1.1) Definition. We say the map $f: M \rightarrow M$ has small displacement if for each $x \in M$ there is a unique minimizing geodesic from $x$ to $f(x)$. Equivalently, $f$ has small displacement if it takes each point into the complement of its cut locus. If $f: M \rightarrow M$ is a diffeomorphism of small displacement we define its displacement vector field $V$ by: if $x \in M$ then $V_{x}$ is the tangent at $x$ to the minimizing geodesic from $x$ to $f(x)$, with $\left|V_{x}\right|=g\left(V_{x}, V_{x}\right)^{1 / 2}=\rho(x, f(x))$.

(1.1.2) Lemma. Let $f: M \rightarrow M$ be a diffeomorphism of small displacement. Then:

(i) the function $\delta_{f}^{2}: M \rightarrow R$ is smooth on $M$,

(ii) $\delta_{f}: M \rightarrow R$ is smooth outside the fixed point set of $f$,

(iii) the displacement vector field $V$ is a smooth vector field on $M$.

Proof. Fix $x \in M$, and let $U=M-$ (cut locus of $x$ ). $U$ is an open cell in $M$, and there is a neighborhood $U_{x}^{\prime} \subset T_{x}(m)$ such that exp: $U_{x}^{\prime} \rightarrow U$ is a diffeomorphism onto $U$. There is a neighborhood $W_{1} \subset U$ containing $x$ such that $f\left(W_{1}\right) \subset U$; and for each $y \in W_{1}$ there is an open set $N_{y} \subset T_{y}(M)$ such that exp: $N_{y} \rightarrow U$ is a diffeomorphism into $U$. We assume $N_{x}=U_{x}^{\prime}$, and we may choose the sets $N_{y}$ so that $W=\bigcup_{W_{1}} N_{v}$ is open in $T(M)$. Then the map $h: W$ $\rightarrow U \times U$ sending $Y \in N_{y}$ to $(y, \exp Y)$ is a diffeomorphism into $U \times U$. Since $N_{x}=U_{x}^{\prime}$, we have $\{x\} \times U \subset h(W)$. The map $U \times U \rightarrow R$ given by $(y, z)$ $\rightarrow \rho(y, z)$ coincides with $\|Y\|$ if $z=\exp Y$ and $Y \in N_{y}$. Now $\|Y\|^{2}$ is differentiable on $W$, so $\rho^{2}(y, z)$ is differentiable on $h(W)$.

Now by the assumption on $f, f(x) \in U$ so $(x, f(x)) \in h(W)$. Since the above argument holds for any $x \in M$, we see that $\delta_{f}^{2}$ is differentiable everywhere on $M$ because it is the composition of differentiable functions. This proves (i), and (ii) follows trivially since $\delta_{f}$ vanishes exactly on the fixed point set of $f$.

Let $Z \subset U$ be an open set with $f(x) \in Z$, and let $W_{0}=f^{-1}(Z) \cap U$. Then the map $f_{0}: W_{0} \rightarrow U \times U$ defined by $f_{0}(y)=(y, f(y))$ is differentiable, and the displacement vector field $V$ restricted to $W_{0}$ is the image of the map $h^{-1} f_{0}: W_{0}$ $\rightarrow T(m)$ which is $C^{\infty}$, since $f_{0}$ is $C^{\infty}$ and $h$ is a diffeomorphism. Since the choice of $x$ is arbitrary, $V$ is $C^{\infty}$ on all of $M$.

(1.1.3) Remark. The displacement function $\delta_{f}$ may fail to be differentiable at a fixed point of $f$ as in the following situation: Let $M=R^{n}, g$ be the 
ordinary metric, and $f$ be the symmetry about the origin 0 sending $R^{n} \ni x$ $\rightarrow-x$. Then $\delta_{f}(x)=2 \sqrt{x_{1}^{2}+\cdots+x_{n}^{2}}$, where $x=\left(x_{1}, \cdots, x_{n}\right)$, and this is not differentiable at $x=0$.

(1.1.4) Definition. (i) For any map $f: M \rightarrow M$ we let Fix $(f)$ denote the set of fixed points of $f$.

(ii) If $f$ has small displacement and is a diffeomorphism, we let Crit $(f)$ denote the set of critical points of $\delta_{f}^{2}$ in $M$.

(1.1.5) Remark. Crit $(f)=$ Fix $(f) \cup\left(\right.$ critical points of $\delta_{f}$ in $M-$ Fix $(f)$ ), since for every $X \in T_{x}(M), X \delta_{f}^{2}=2 \delta_{f}(x) X \delta_{f}$ whenever $\delta_{f}$ is differentiable.

(1.2) Suppose now that $f: M \rightarrow M$ is an isometry of small displacement. We wish to differentiate $\delta_{f}$. To do this fix $x \in M-$ Fix $(f)$, and let $X \in T_{x}(M)$ be any non-zero vector, and $b(s)$ a smooth curve through $x$ with tangent $X$ at $x=b(0)$. Then $X \delta_{f}=\left.\frac{d}{d s}\right|_{s=0} \rho(b(s), f(b(s)))$. Let $a=\rho(x, f(x))$. By assumption on $x, a>0$. The displacement vector field $V$ is $C^{\infty}$, so we have a $C^{\infty}$ map $Q:[0, a] \times[0, \infty) \rightarrow M$ given by $Q(s, t)=\exp _{b(s)}\left(t \frac{V}{a}\right)$. Here we may take $t$ and $s$ in slightly larger open intervals to avoid one-sided derivatives. For fixed $s=s_{0}$ the curve $Q\left(s_{0}, t\right)$ is the unique minimizing geodesic from $b\left(s_{0}\right)$ to $f\left(b\left(s_{0}\right)\right)$, and is parametrized proportional to arc-length.

Let $T=Q_{*} \partial / \partial t$ and $X=Q_{*} \partial / \partial s$; these are $C^{\infty}$ vector fields on the image of $Q$, and have the two properties: $[T, X]=0$ and $\nabla_{T} T=0$. The first follows from $[T, X]=\left[Q_{*} \partial / \partial s, Q_{*} \partial / \partial t\right]=Q_{*}[\partial / \partial t, \partial / \partial s]=0$, and the second holds because $T$ is the tangent field to a family of geodesics. Moreover, if $b(s)$ is a geodesic then $\nabla_{X} X=0$ when $t=0$ or $a$ since $f(b(s))$ is also a geodesic. Evidently $g(T, T)$ is independent of $t$, and we let $C(s)=\sqrt{ } g(T, T)$.

(1.2.1) Theorem. Let $f: M \rightarrow M$ be an isometry of small displacement and $x \in M$. Then $x \in \mathrm{Crit}(f)$ if and only if $f$ preserves the minimizing geodesic from $x$ to $f(x)$.

Proof. Let $c$ be the minimizing geodesic from $x$ to $f(x)$ and assume $x \notin \operatorname{Fix}(f)$. Then

$$
\rho(b(s), f(b(s)))=\int_{0}^{a} \sqrt{g(T, T)}(s, t) d t,
$$

so

$$
\begin{aligned}
X_{b(s)} \delta_{f} & =\frac{d}{d s} \rho(b(s), f(b(s)))=\int_{0}^{a} \partial / \partial s \sqrt{g(T, T)} d t \\
& =\frac{1}{C(s)} \int_{0}^{a} g\left(\nabla_{X} T, T\right) d t=\frac{1}{C(s)} \int_{0}^{a} g\left(\nabla_{T} X, T\right) d t
\end{aligned}
$$




$$
\begin{aligned}
& =\frac{1}{C(s)} \int_{0}^{a} \partial / \partial t g(X, T) d t \\
& =\frac{1}{C(s)}(g(X, T)(s, a)-g(X, T)(s, 0)) .
\end{aligned}
$$

Here we have used $\nabla_{X} T-\nabla_{T} X=[X, T]=0$, and $\nabla_{T} T=0$. Thus $X_{x} \delta_{f}=$ $g(X, T)(0, a)-g(X, T)(0,0)$, since $C(0)=1$. If $c$ is normal to $b(s)$ at $x$, then $g(X, T)(0,0)=0$, which shows that $x \in$ Crit $(f)-$ Fix $(f)$ implies $g(X, T)(0, a)$ $=0$. Now by definition of $X, X_{f(b(0))}=f_{*} X_{b(s)}$, so $x \in$ Crit $(f)-$ Fix $(f)$ implies that $f$ preserves the normal space to $c$; this is equivalent to $f$ preserving $c$.

Now suppose $f$ preserves the geodesic $c$. If $X \in T_{x}(M)$ is tangent to $c$, then $X \delta_{f}=0$ because $\delta_{f}$ is measured along $c$ for all points on $c$. Thus, if $X$ is any vector in $T_{x}(M)$, then $X \delta_{f}=X_{0} \delta_{f}$ where $X_{0}$ is the component of $X$ normal to $c$. But then $X_{0} \delta_{f}=g\left(X_{0}, T\right)(0, a)=0$, since if $f$ preserves $c$ it must also preserve the normal space to $c$. This shows that $X \delta_{f}=0$ for all $X \in T_{x}(M)$, so $x \in$ Crit $(f)$. The theorem holds vacuously at every fixed point of $f$.

(1.2.2) Remark. By " $f$ preserves the geodesic" we mean that $f$ restricted to the geodesic is a simple translation along the geodesic. This excludes a reflection about some isolated fixed point.

(1.3) We now compute the second derivative of $\delta_{f}$. Let $x \in M-$ Fix $(f)$, $b(s)$ be a geodesic with $b(0)=x$, and $X$ be defined as before. In particular, $X_{b(s)}$ is the tangent to $b(s)$ and $X_{f(b(s))}$ is the tangent to $f(b(s))$. Then

$$
\begin{aligned}
X_{b(\alpha)}^{2} \delta_{f} & =\frac{d^{2}}{d s^{2}} d(b(s), f(b(s)))=\int_{0}^{a} \frac{\partial^{2}}{\partial s^{2}} \sqrt{g(T, T)} d t \\
& =\int_{0}^{a} \frac{g(T, T)\left(g\left(\nabla_{X} \nabla_{X} T, T\right)+g\left(\nabla_{X} T, \nabla_{X} T\right)\right)-g\left(\nabla_{X} T, T\right)^{2}}{g(T, T)^{3 / 2}} d t
\end{aligned}
$$

Now $[X, T]=0$ implies that $\nabla_{X} T=\nabla_{T} X$, and

$$
\nabla_{X} \nabla_{T} X=\nabla_{T} \nabla_{X} X+R(X, T) X,
$$

so

$$
g\left(\nabla_{X} \nabla_{X} T, T\right)=\frac{\partial}{\partial t} g\left(\nabla_{X} X, T\right)+g(R(X, T) X, T)
$$

Moreover, $g(R(X, T) X, T)=-K(X, T)\left(g(T, T) g(X, X)-g(X, T)^{2}\right)$, so,

$$
\begin{aligned}
X_{b(s)}^{2} \delta_{f}= & \frac{1}{C(s)^{3}} \int_{0}^{a}\left\{g ( T , T ) \left(\frac{\partial}{\partial t} g\left(\nabla_{X} X, T\right)-K(X, T)\right.\right. \\
& \left.\times\left(g(T, T) g(X, X)-g(X, T)^{2}\right)+g\left(\nabla_{X} T, \nabla_{X} T\right)-g\left(\nabla_{X} T, T\right)^{2}\right\} d t
\end{aligned}
$$




$$
\begin{aligned}
& =\frac{1}{C(s)} \int_{0}^{a} \frac{\partial}{\partial t} g\left(\nabla_{X} X, T\right) d t \\
& +\frac{1}{C(s)^{3}} \int_{0}^{a}\left\{g(T, T) g\left(\nabla_{X} T, \nabla_{X} T\right)-g\left(\nabla_{X} T, T\right)^{2}\right\} d t \\
& \left.\quad-K(X, T)\left(g(T, T) g(X, X)-g(X, T)^{2}\right)\right\} .
\end{aligned}
$$

Since $b(s)$ is a geodesic, $\nabla_{X} X=0$ at both $t=0$ and $t=a$. Therefore,

$$
\int_{0}^{a} \partial / \partial t g\left(\nabla_{X} X, T\right) d t=g\left(\nabla_{X} X, T\right)(s, a)-g\left(\nabla_{X} X, T\right)(s, 0)=0
$$

and

$$
\begin{aligned}
X_{b(s)}^{2} \delta_{f}= & \frac{1}{C(s)^{3}} \int_{0}^{a}\left\{g(T, T) g\left(\nabla_{X} T, \nabla_{X} T\right)-g\left(\nabla_{X} T, T\right)^{2}\right. \\
& \left.-K(X, T)\left(g(T, T) g(X, X)-g(X, T)^{2}\right)\right\} d t
\end{aligned}
$$

Here $K(X, T)$ is the curvature of the 2-plane spanned by $X$ and $T$. This equation is valid even when $x \notin$ Crit $(f)$.

Note. A subset $S$ of a Riemannian manifold $M$ is said to be locally convex, if for every pair of points $x, y \in S$, which are sufficiently close, the minimizing geodesic from $x$ to $y$ lies is $S$.

(1.3.2) Lemma. Let $M$ be a complete connected Riemannian manifold, and $S \subset M$ a closed, connected and locally convex subset. Then $S$ is a totally geodesic submanifold of $M$ with possibly non-empty boundary. (Here we do not assume the boundary is smooth or of codimension one.)

Proof. Let $x \in S$, and $N_{x}$ be a convex normal neighborhood of $x$ in $M$. For the moment we restrict to $N_{x}$. Suppose $y \in S \cap N_{x}$ and $y \neq x$. Then the geodesic segment $\gamma$ from $x$ to $y$ lies in $S$. Choose any interior point $z_{0}$ of $\gamma$ and a ball $B_{r_{1}}\left(z_{0}\right)$ with radius $r_{1}=\min \left\{d\left(x, z_{0}\right), d\left(y, z_{0}\right)\right\}$ and center $z_{0}$. Suppose $B_{r_{1}}\left(z_{0}\right) \cap S \not \subset \gamma$ and $z_{2} \in B_{r_{1}}\left(z_{0}\right) \cap S-\gamma$. We construct a cone $\Delta_{2}$ over $B_{r_{1}}\left(z_{0}\right) \cap \gamma$ with vertex $z_{2}$ and generators the geodesics from $z_{1}$ to the points of $B_{r_{1}}\left(z_{0}\right) \cap \gamma$. By the assumption on $z_{2}, \Delta_{2}$ is a two-dimensional cell with boundary. Again choose an interior point $z_{2}$ of $\Delta_{2}$ and let $r_{2}=\inf \left\{\mathrm{d}\left(z_{2}, w\right) \mid w \in \partial \Delta_{2}\right\}$. Suppose $Z_{3} \in B_{r_{2}}\left(z_{2}\right) \cap S-\Delta_{2}$, and construct the cone $\Delta_{3}$ over $\Delta_{2} \cap B_{r_{2}}\left(z_{2}\right)$ with vertex $z_{3}$ and geodesic generators. By choosing a possibly smaller $r_{2}$ we can make sure that the generators of $\Delta_{3}$ are always transverse to $\Delta_{2} \cap B_{r .}\left(z_{2}\right)$. Then the cone $\Delta_{3}$ is a three-dimensional cell with boundary. We continue in this manner, and must eventually stop since $\operatorname{dim} M<\infty$. Say the last cone constructed is $\Delta_{k}$. Choose an interior point $z_{k} \in \Delta_{k}$; it is clear from the convexity of $S$ that there is a geodesic segment from $z_{k}$ to each point of $\Delta_{k}$ which lies in $S$ (in fact, it lies in $\Delta_{k} \subset S$ since otherwise we could have constructed $\left.\Delta_{k+1}\right)$. This means 
the interior of $\Delta_{k}$ is a convex neighborhood in $S$ and therefore a totally geodesic submanifold (with boundary) of $M$. Such a submanifold has the property that each of its points $w$ is contained in a convex normal neighborhood, which in this case is the image by the exponential map of a ball in some linear $k$ dimensional subspace of $T_{w}(M)$. We make the above construction for all choices of points in the interior of $S$, and compare nearby normal neighborhoods in $S$. We claim they must all have dimension $k$. This is seen by choosing a point in one of the neighborhoods $U_{1}$ which does not lie in the other neighborhood $U_{2}$ (we assume $\operatorname{dim} U_{2}=k$ ). This point is then the vertex of a cone over a $k$-dimensional normal coordinate ball in $N_{2}$, and by maximality of the dimension of $U_{2}$ we must get back a $k$-dimensional cell. This implies $\operatorname{dim} U_{1}=k$. Then by connectedness of $S$, we see that every ball in $S$ has dimension $k$. The intersection of convex balls is a convex neighborhood, so the interior of $S$ is in fact a $k$ dimensional totally geodesic submanifold.

(1.3.3) Remark. It may happen that $\partial$ Crit $(f) \neq \phi$, as seen by the following example: We consider the Euclidean plane $R^{2}$ with the usual coordinates $(x, y)$. Let $\varepsilon>0$. Then there is a $C^{\infty}$ function $\varphi(y)$ on $R^{1}$ with the property that $\varphi(y)=1 / y^{2}$ if $y>\varepsilon, \varphi(y) \equiv 1$ if $y<0$, and $\varphi(y)>0$ everywhere. Then let $d s^{2}=\varphi(y)\left(d x^{2}+d y^{2}\right)$ be the Riemannian metric. On the set $\{(x, y) \mid y>\varepsilon\}, d s^{2}$ is the metric of the hyperbolic plane (Poincaré upper half-space); and on $\{(x, y) \mid y<0\}, d s^{2}$ is the usual Euclidean metric. If we let $a>0$ be a small number then the map $f: R^{2} \rightarrow R^{2}$ given by $f(x, y)=(x+a, y)$ is an isometry of the set $R^{2}$ considered as a Riemannian manifold with metric $d s^{2} . f$ has small displacement and $\{(x, y) \mid y>0\} \subset$ Crit $(f)$ since $f$ has constant displacement on this set. However, $\{(x, y) \mid y>\varepsilon\} \cap$ Crit $(f)=\emptyset$ since the displacement in $\{(x, y) y>\varepsilon\}$ is decreasing in $y$. Therefore $\partial$ Crit $(f) \neq \emptyset$.

(1.3.4) Theorem. Let $M$ have curvature $K \leq 0$, and assume $f: M \rightarrow M$ is an isometry of small displacement. Then

(i) Crit $(f)$ is a totally geodesic submanifold possibly with boundary,

(ii) $\delta_{f}$ takes its absolute minimum on Crit $(f)$.

(iii) If Fix $(f)=\emptyset$, then Crit $(f)$ is connected; if Fix $(f) \neq \emptyset$, then Crit $(f)$ $=$ Fix $(f)$.

(iv) If $M$ is simply connected, then Fix ( $f)$ is connected.

(v) If $K<0$ and $\operatorname{Fix}(f)=\emptyset$, then Crit $(f)$ is either empty or consists of a single geodesic.

Proof. Under the curvature assumption, we have $X_{b(s)}^{2} \delta_{f} \geq 0$ for every geodesic $b(s)$ by the following:

$$
\begin{gathered}
g(T, T) g\left(\nabla_{X} T, \nabla_{X} T\right) \rightarrow g\left(\nabla_{X} T, T\right)^{2} \geq 0, \\
g(T, T) g(X, X)-g(X, T)^{2} \geq 0
\end{gathered}
$$

by the Cauchy-Schwarz inequality. Thus the right side of equation (1.3.1) is non-negative, and hence $X_{b(s)}^{2} \delta_{f} \geq 0$ whenever $b(s) \notin$ Fix $(f)$. Suppose now that 
$x \in$ Crit $(f)-$ Fix $(f)$, and let $b(s)$ be any geodesic with $b(0)=x$. Suppose $b\left(s_{0}\right)$ is the first point on $b$, which lies in Fix $(f)$. Then $X_{b(s)} \delta_{f}$ is non-decreasing along $b(s)$, so that in fact $\delta_{f}$ is non-decreasing along $b(s)$ because $X_{b(0)} \delta_{f}=0$. But this is impossible since $\delta_{f}(x)>0$ and $\left.\delta_{f}\left(b s_{0}\right)\right)=0$. Thus either Crit $(f)$ - Fix $(f)=\emptyset$ or Fix $(f)=\emptyset$. This proves the second part of (iii). Let Fix $(f)$ $=\emptyset$. Then the above argument shows that if $b\left(s_{0}\right) \in C$ rit $(f)$, then $X_{b(s)} \delta_{f}=0$ for all $s \in\left[0, s_{0}\right]$. This means that $\delta_{f}$ is constant on Crit $(f)$. The condition $X_{b(s)}^{2} \delta_{f} \geq 0$ shows that each point of Crit $(f)$ is a relative minimum of $\delta_{f}$, so that in fact it must be an absolute minimum. If Fix $(f) \neq \emptyset$, then Crit $(f)=$ Fix $(f)$, so that again $\delta_{f}$ takes its absolute minimum on Crit $(f)$, and hence (ii) is proved.

Now if Fix $(f) \neq \emptyset$, then we take $x, y \in$ Fix $(f)$, which lie in the same component of Fix $(f)$ and are sufficiently close so there is a unique minimizing geodesic $c$ between them. Thus $f(c)$ is a geodesic of the same length between them, so in fact $c=f(c)$. Moreover, $c \subset$ Fix $(f)$, and Fix $(f)$ is totally geodesic. If Fix $(f)=\emptyset$, choose $x, y \in$ Crit $(f)$, and let $b(s)$ be any geodesic between them with $b(0)=x$ and $b\left(s_{0}\right)=y$. Now $\delta_{f}$ is constant along $b(s)$, and $\delta_{f}$ takes its absolute minimum at $x$ and $y$, so all points on $b(s)$ between $x$ and $y$ lie in Crit $(f)$. This proves (i) by Lemma 1.3.2, and also proves the first part of (iii). (iv) follows froms the fact that in a simply connected manifold with curvature $K<0$ there are no cut points, so every pair of points is connected by a unique minimizing geodesic, and the above argument for Fix $(f) \neq \emptyset$ applies.

Now assume that $K<0$ everywhere on $M$, and $x \in$ Crit $(f)-$ Fix $(f)$. If $b(s)$ is a geodesic transverse to the minimizing geodesic $c$ from $x$ to $f(x)$, then we have $g(T, T) g(X, X)-g(X, T)^{2}>0$ at $s=0$ and $t=0$ or $a$, since

$$
\begin{aligned}
& g(T, T) g(X, X)-g(X, T)^{2} \\
& \quad=g(T, T) g(X, X)\left(1-\cos ^{2}(\text { angle between } X \text { and } T)\right) .
\end{aligned}
$$

Thus $X_{b(s)}^{2} \delta_{f}>0$ at $s=0$ so that $X_{b(s)} \delta_{f}>0$ for $s$ near 0 . This means $\delta_{f}$ is strictly increasing along $b(s)$, so $b(s)$ cannot lie in Crit $(f)$. Since $c$ is evidently in Crit $(f)$ the conclusion follows.

(1.3.5) Corollary. If $M$ is simply connected and $K \leq 0$, then the results of the above theorem hold for any isometry.

(1.3.6) Remark. If $M$ is an analytic manifold and has curvature $K \leq 0$, then Crit $(f)$ is a real analytic submanifold, which is totally geodesic and has no boundary. The fact that Crit $(f)$ has no boundary follows since if an interval of a geodesic $\gamma$ lies in Crit $(f)$, then the whole geodesic $\gamma$ must lie in Crit $(f)$ because $\delta_{f}^{2}$ is then an analytic function $\gamma$ whose derivative is zero in an interval and hence zero everywhere. If there were a boundary point $x$, there would have to be a geodesic starting inside Crit $(f)$ and leaving through $x$, contradicting the fact that $\gamma$ must lie in Crit $(f)$. Note that if $M$ is analytic, then every isometry $f: M \rightarrow M$ is analytic and the displacement function $\delta_{f}^{2}$ for isometries of small displacement is also analytic. 
(1.3.7) Theorem. Let $M$ be any complete connected Riemannian manifold, and $f: M \rightarrow M$ an isometry of small displacement. If $h: M \rightarrow M$ is any isometry, then Crit $\left(h \cdot f \cdot h^{-1}\right)=h($ Crit $(f))$. That is, $h(x) \in$ Crit $(f)$ if and only if $x \in \operatorname{Crit}\left(h^{-1} \circ f \circ h\right)$.

Proof. $h(x) \in$ Crit $(f)$ if and only if $f$ preserves the minimizing geodesic $c$ from $h(x)$ to $f h(x)$. Let $c(0)=h(x), c(a)=f h(x)$ with $a=d(h(x)$, $f h(x))$. Then $f$ preserves $c$ if and only if $f^{2} h(x)=c(2 a)$. Now $f^{2} h(x)=c(2 a)$ when $h^{-1} f^{2} h(x)$ $=h^{-1} c(2 a)$. The geodesic $h^{-1} c$ is the minimizing geodesic from $x$ to $h^{-1} f h(x)$, so $x \in$ Crit $\left(h^{-1} f h\right)$ if and only if $\left(h^{-1} f h\right)^{2} x=h^{-1} c(2 a)$. But $\left(h^{-1} f h\right)^{2}=h^{-1} f^{2} h$, so the result follows.

(1.3.8.) Theorem. Suppose $M$ has curvature $K \leq 0$ and $f$ is an isometry of small displacement. Let $x \in$ Crit $(f)$ - Fix $(f), b(s)$ be any geodesic in Crit $(f)$, which is transverse to the displacement vector field at $x(b(0)=x), V$ be the displacement vector field of $f$ along $b(s)$, and $a=\delta_{f}(x)$. Then the surface $Q$ defined by $Q(s, t)=\exp _{b(s)}(t V / a)$ has curvatuae $K \equiv 0$, and the vector fields $T=Q_{*} \partial / \partial t$ and $X=Q_{*} \partial / \partial s$ are parallel on $Q$.

Proof. We know $X_{b(s)}^{2} \delta_{f}=0$ since $\delta_{f}$ is constant along $b(s)$, so

$$
\begin{aligned}
\int_{0}^{a} & \left\{\left(g(T, T) g\left(\nabla_{X} T, \nabla_{X} T\right)-g\left(\nabla_{X} T, T\right)^{2}\right)\right. \\
& \left.-K(X, T)\left(g(T, T) g(X, X)-g(X, T)^{2}\right)\right\} d t=0 .
\end{aligned}
$$

Since $b(s)$ is transverse to the geodesic $c$ between $x$ and $f(x), g(T, T) g(X, X)$ $-g(X, T)^{2}>0$, so we must have $K(X, T)=0$ for all $s$ and $t$. Furthermore, the curves $Q(s, t)$ for either $s$ or $t$ constant are then a Euclidean coordinate system in $Q$, so their tangents form parallel vector fields.

(1.3.9) Theorem. Let $M$ be a Riemannian manifold, $X$ a Killing vector field on $M$, and $g_{t}$ its 1-parameter group of isometries, and assume $g_{t}$ has small displacement for $t \in[0,1]$. Then Crit $\left(|X|^{2}\right)=\bigcap_{n=1}^{\infty} \operatorname{Crit}\left(g_{1 / n}\right)$ and Crit $\left(g_{1 /(n+1) !}\right) \subset$ Crit $\left(g_{1 / n !}\right)$ for all $n=1,2, \ldots$.

Proof. It is clear that Crit $\left(|X|^{2}\right) \subset$ Crit $\left(g_{t}\right)$ for all $t \in(0,1]$ since Crit $\left(|X|^{2}\right)$ $=\left\{x \in M \mid g_{t} x\right.$ is a geodesic $\}$. Suppose $x \in \bigcap_{n=1}^{\infty}$ Crit $\left(g_{1 / n !}\right)$. Since Crit $\left(g_{1 / n}\right) \subset$ Crit $\left(g_{1}\right)$ for all $n$, the geodesic preserved by $g_{1 / n}$ is the same as that for $g_{1}$, and therefore the orbit $g_{t} x$ crosses the geodesic $c$ from $x$ to $g x$ at the points $g_{m / n !} x$ for $1 \leq m \leq n !$. The set of points $\left\{g_{m / n !} \mid 1 \leq m \leq n\right.$ !, all $\left.n\right\}$ is dense on $c$, so in fact $g_{t} x=c$. The fact that Crit $\left(g_{1 /(n+1) !}\right) \subset$ Crit $\left(g_{1 / n !}\right)$ is obvious from Theorem 1.2.1.

(1.3.10) Corollary. Let $M$ be analytic, and suppose its curvature $K$ is non-positive. Let $X$ be a Killing vector field, and $g_{t}$ its 1-parameter group, and assume $g_{t}$ has small displacement for all $t \in[0,1]$. Then there is a $t_{0} \in(0,1]$ such that Crit $\left(|X|^{2}\right)=\operatorname{Crit}\left(g_{t_{0}}\right)$. 
Proof. If $X=0$ at some point, then $q_{t}$ has a fixed point and the corollary follows from Theorem 1.3.3 (iii). Suppose $X \neq 0$ everywhere. Then each Crit $\left(g_{t}\right)$ is a connected submanifold of $M$ without boundary (Remark 1.3.5). Let $k_{n}=\operatorname{dim}$ Crit $\left(g_{1 / n !}\right)$. Since the critical sets Crit $\left(g_{1 / n !}\right)$ are nested and converge to Crit $\left(|X|^{2}\right)$, we must have $k_{n} \rightarrow \operatorname{dim} \operatorname{Crit}\left(|X|^{2}\right)$, which means that for some $n, k_{n}=\operatorname{dim}$ Crit $\left(|X|^{2}\right)$. Then Crit $\left(|X|^{2}\right)$ is a connected submanifold of the connected manifold Crit $\left(g_{1 / n !}\right)$, and they must be equal since they have the same dimension.

\section{Chapter II. Homogeneous and symmetric spaces}

We now assume that $M=G / K$ is a reductive homogeneous space which is connected and has normal metric in which it is complete. We fix a direct sum decomposition

$$
\boldsymbol{G}=\boldsymbol{K}+\boldsymbol{m}
$$

of the Lie algebra $G$ of $G$, where $K=$ Lie algebra of $K$, and $m$ is a complementary subspace with the property that ad $(K) m \subset m$. We consider only those isometries of $M$ coming from elements of $G$.

(2.1) Let $g \in G$ be an isometry of small displacement, and let $x \in M$. We assume $x$ is identified with the identity coset of its isotropy group $K$. Then there is a unique shortest $T \in m$ such that $g x=(\exp T) x$, and $(\exp t T) x, 0 \leq t$ $\leq 1$, is the minimizing geodesic from $x$ to $g x$. Thus $(\exp -T) g x=x$ so that $k=(\exp -T) g \in K$, and we have a unique decomposition $g=(\exp T) k$.

(2.1.1) Theorem. $x(=K)$ is in Crit $(g)$ if and only if ad $(k)=T$, where $g=(\exp T) k$ in the above decomposition.

Proof. By Theorem 1.2.1, $x \in$ Crit $(g)$ if and only if $g$ preserves the geodesic $(\exp t T) x$. This is true exactly when $g(\exp t T) x=(\exp (1+t) T) x$. Now $g(\exp T) x=(\exp (1+t) T) x$ if and only if $(\exp -t T) k(\exp t T) x=x$; that is, when $(\exp -t T) k(\exp t T) \in K$ for all $t$. This curve has tangent $d L_{k}(T)-d R_{k}(T)$ $=T-\operatorname{ad}(k) T$ at $t=0$, where $L_{k}$ (resp. $R_{k}$ ) is the left (resp. right) translation by $k$. Since $\operatorname{ad}(K) m \in m$, we have $\operatorname{ad}(k) T \in m$ so the tangent lies in $m$. Since it must also lie in $K$, it must vanish; that is, $\operatorname{ad}(k) T=T$.

Conversely, if ad $(k) T=T$ then

$$
\begin{aligned}
g(\exp t T) x & =(\exp T) k(\exp t T) k^{-1} x=(\exp T)(\exp t \operatorname{ad}(k) T) x \\
& =(\exp T)(\exp t T) x=(\exp (1+t) T) x
\end{aligned}
$$

So $g$ preserves the geodesic $(\exp t T) x$ from $x$ to $g x$.

(2.1.2) Corollary.

$$
\text { Crit }(g)=\left\{h x \mid \operatorname{ad}\left(k_{h}\right) T_{h}=T_{h}, h^{-1} g h=\left(\exp T_{h}\right) k_{h}, h \in G\right\},
$$

where $h^{-1} g h=\left(\exp T_{h}\right) k_{h}$ is the unique decomposition of Theorem 2.1.1. 
Proof. Clearly $h^{-1} g h$ has small displacement if $g$ does, so the proof follows from Theorems 1.3.7 and 2.1.1.

(2.2) Let $M=G / K$ be a compact connected Riemannian homogeneous space with normal metric. Assume $G$ is compact and semi-simple, so that the Killing form $B$ is negative definite on $\boldsymbol{G}$ and is invariant under the adjoint action of $\boldsymbol{G}$. Let $\boldsymbol{G}=\boldsymbol{K}+\boldsymbol{m}$ as usual, with $\boldsymbol{K}$ and $m$ orthogonal by $-\boldsymbol{B}$.

(2.2.1) Lemma. There is a number $r>0$ such that if $g \in \exp B, B_{r}=$ $\left\{Y \in G \mid(-B(Y, Y))^{1 / 2}<r\right\}$, then $g=(\exp T)(\exp S)$ for unique shortest $T \in m$, $S \in K$; and $(\exp S)(\exp T)=(\exp T)(\exp S)$ if and only if $[T, S]=0$.

Proof. Define a map $K \times m \stackrel{\varphi}{\longrightarrow} G$ by $\varphi(S, T)=(\exp T)(\exp S) . \varphi$ is clearly regular at $(0,0)$ and is differentiable everywhere. Then by the inverse function theorem there is a neighborhood of $(0,0)$ in $K \times m$ on which $\varphi$ is a diffeomorphism. Let $r_{0}>0$ be maximal for the property that $\exp : G \rightarrow G$ is a diffeomorphism on $B_{r_{0}}=\left\{Y \in G \mid-B(Y, Y)<r^{2}\right\}$. Let $V_{1}=K \cap B_{r_{0}}, V_{2}$ $=m \cap B_{r_{0}}$, and $V \subset V_{1} \times V_{2}$ be the maximal neighborhood of the form $V=\varphi^{-1}\left(\exp \left(B_{r}\right)\right)$ on which $\varphi$ is a diffeomorphism. It is clear that $r>0$.

Suppose now that $g \in \exp B_{r}$; then $g$ is written uniquely as $g=(\exp T)(\exp S)$ for $T \in m, S \in K$. Assume $(\exp T)(\exp S)=(\exp S)(\exp T)$, which means $\exp \operatorname{ad}(\exp S) T=\exp T$. But since $B_{r}$ is $\operatorname{ad}(G)$-invariant, we have $\operatorname{ad}(\exp S) T$, $T \in B_{r}$ so that $\operatorname{ad}(\exp S) T=T$ as $\exp$ is a diffeomorphism on $B_{r} \subset B_{r_{0}}$. Similarly, $\operatorname{ad}(\exp T) S=S$, which means $(\exp S)(\exp t T)=(\exp t T)(\exp S)$ for all $t$, Applying the above argument to $t T$ and $S$, for any $t \in[0,1]$, we get $(\exp t S)(\exp t T)=(\exp t T)(\exp t S)$, which is equivalent to $[T, S]=0$. It is obvious that $[T, S]=0$ implies $(\exp T)(\exp S)=(\exp S)(\exp T)$.

(2.2.2) Theorem. Let $M=G / K$ be a compact homogeneous space with normal metric, and assume $G$ is compact semisimple. Let $X \in B_{r}, g=\exp X$ be the associated isometry, and $x=K$. Then $h x \in \operatorname{Crit}(g)$ for $h \in G$ if and only if $h^{-1} g h=(\exp T)(\exp S)$ with $[T, S]=0$, where $S=\left(\operatorname{ad}\left(h^{-1}\right) X\right)_{K}, T=$ $\left(\operatorname{ad}\left(h^{-1}\right) X\right)_{m}$, and $g=\exp X$.

Proof. We know that $h x \in \operatorname{Crit}(g)$ if and only if $h^{-1} g h=(\exp T) k$ for $T \in m$, $k \in K$ where $\operatorname{ad}(k) T=T$. Here there is no question of uniqueness of $T$ since $B_{r}$ is $\operatorname{ad}(G)$-invariant and $\varphi$ is a diffeomorphism on $\exp B_{r}$. Thus $h^{-1} g h \in \exp B_{r}$; if $\operatorname{ad}(k) T=T$ then $(\exp T) k=k(\exp T)$, and since $k=\exp S$ Lemma 2.2.1 shows that $[S, T]=0$. In this case $(\exp T)(\exp S)=\exp (S+T)=h^{-1}(\exp X) h$, so that $S=\left(\operatorname{ad}\left(h^{-1}\right) X_{K}\right.$ and $T=\left(\operatorname{ad}\left(h^{-1}\right) X_{m}\right.$. Conversely, if $[S, T]=0$ then obviously $\operatorname{ad}(k)=T$.

(2.2.3) Corollary. Let $M=G / \dot{K}$ be a connected symmetric space of compact type, with $\sigma$ the symmetry in both $G$ and $G$, and let $x=K$. If $g \in \exp B_{r}$ as in Theorem 2.2.2, then Crit $(g)=\left\{h^{-1} x \mid h \in G\right.$ and $\left.[\operatorname{ad}(h) X, \sigma \operatorname{ad}(h) X]=0\right\}$; $g=\exp X$.

Proof. For any $Y \in G,[Y, \sigma Y]=\left[Y_{K}+Y_{m}, Y_{K}-Y_{m}\right]=2\left[Y_{K}, Y_{m}\right]$, so the result follows from Theorem 2.2.2. 
(2.2.4) Corollary. Under the assumptions in Theorem 2.2.2, Crit $\left(|X|^{2}\right)=$ Crit $\left(g_{t}\right)$ where $g_{t}=\exp t X$ and $t \in(0,1]$.

Proof. Clearly Crit $\left(|X|^{2}\right) \subset$ Crit $\left(g_{t}\right)$ for each $t \in(0,1]$. Conversely, if for any $t \in(0,1],\left[(t X)_{K},(t X)_{m}\right]=0$, then this is true for all $t \in(0,1]$. This does not depend on the choice of decomposition $G=K+m$; therefore, $x \in \operatorname{Crit}\left(g_{t_{1}}\right)$ if and only if $x \in$ Crit $\left(g_{t_{2}}\right)$. Since $\bigcap_{0<t \leq 1}$ Crit $\left(g_{t}\right)=$ Crit $\left(|X|^{2}\right)$ the result follows.

(2.3) In this section we assume $M=G / K$ is a connected Riemannian symmetric space of compact type, and $g \in \exp B_{r}$ an isometry having $x=K$ in Crit (g) with $g=\exp X$.

(2.3.1) Lemma. If $X \in G$ is such that $[X, \sigma X]=0$, then there is a Cartan subalgebra $\mathfrak{h}$ of $\boldsymbol{G}$ such that $X \in \mathfrak{h}$ and $\sigma \mathfrak{h}=\mathfrak{h}$.

Proof. Let $X_{m}=\frac{1}{2}(X-\sigma X)$ and $X_{K}=\frac{1}{2}(X+\sigma X)$ so that $X_{m} \in m, X_{K} \in K$. Let $Z_{G}\left(X_{K}\right)=$ centralizer of $X_{K}$ in $G$. $\sigma$ is the identity on $K$, so if $Y \in Z_{G}\left(X_{K}\right)$ then $\left[\sigma Y, X_{K}\right]=\left[\sigma Y, \sigma X_{K}\right]=\sigma\left[Y, X_{K}\right]=0$. Therefore $\sigma Z_{G}\left(X_{K}\right)=Z_{G}\left(X_{K}\right)$, and $Z_{G}\left(X_{K}\right)=Z_{K}\left(X_{K}\right)+Z_{m}\left(X_{K}\right)$. Since $[X, \sigma X]=0,\left[X_{m}, X_{K}\right]=0$, so $X_{m} \in Z_{m}\left(X_{K}\right)$. Choose $A \subset Z_{m}\left(X_{K}\right)$ a maximal abelian subspace containing $X_{m}$, and let $B \subset$ (centralizer of $A$ in $\left.Z_{K}\left(X_{K}\right)\right)$ be a maximal abelian subspace necessarily containing $X_{K}$. It is clear that $A$ and $B$ are non-empty since $X_{m} \in A$ and $X_{K} \in B$. The subspace $A+B$ of $G$ is an abelian subalgebra which is invariant under $\sigma$. Suppose $Y \in G$ commutes with every element of $A+B$. If we let $Y=Y_{K}+Y_{m}$ with $Y_{K} \in K, Y_{m} \in m$, then $[Y, A]=0=[Y, B]$ implies $\left[Y_{K}, A\right]=0=\left[Y_{K}, B\right]$, and $\left[Y_{m}, A\right]=0=\left[Y_{m}, B\right]$. Since $A$ is maximal abelian in $Z_{m}\left(X_{K}\right), Y_{m} \in A$. $Y_{K}$ centralizes $A$ and also $B$, so by maximality of $B, Y_{K} \in B$, and $Y=Y_{K}+Y_{m} \in A+B$. Thus $A+B$ is a maximal abelian subalgebra of $\boldsymbol{G}$, and is a Cartan subalgebra, since $G$ is compact.

(2.3.2) Theorem. Let $M=G / K$ be a connected symmetric space of compact type, and $g \in \exp B_{r}$ an isometry. If $x \in \mathrm{Crit}(g)$, then the component of Crit $(g)$ containing $x$ is $Z_{G}^{0}(g) \cdot x$. Here $Z_{G}^{0}(g)$ is the identity component of the centralizer $Z_{G}(g)$ of $g$ in $G$.

Proof. By Corollary 2.2.3, $h^{-1} x \in \mathrm{Crit}(g)$ if and only if $[\operatorname{ad}(h) X, \sigma \operatorname{ad}(h) X]$ $=0$. It suffices to consider only those $h \in \exp m$ since $M$ is complete.

Let $\mathfrak{h}_{i}$ be the distinct Cartan subalgebras of $G$ which contain $X$, and choose regular elements $X_{i} \in \mathfrak{h}_{i}$ which lie in $B_{r}$. This is possible since $t X_{i}$ is regular when $X_{i}$ is regular and $t \neq 0$. Now for any $h \in G, \operatorname{ad}(h) \mathfrak{h}_{i}$ are the distinct Cartan subalgebras containing $\operatorname{ad}(h) X$, so if $h^{-1} x \in$ Crit $(g)$ then by Lemma 2.3.1 there is an index $i$ such that $\sigma \operatorname{ad}(h) \mathfrak{h}_{i}=\operatorname{ad}(h) \mathfrak{h}_{i}$. In particular, this means that $\left[\operatorname{ad}(h) X_{i}, \sigma \operatorname{ad}(h) X_{i}\right]=0$, so $h^{-1} x \in C$ rit $\left(\exp X_{i}\right)$. Conversely, if $h^{-1} x \in$ Crit $\left(\exp X_{i}\right)$ then $\left[\operatorname{ad}(h) X_{i}, \sigma \operatorname{ad}(h) X_{i}\right]=0$. Now $\operatorname{ad}(h) X_{i}$ and $\sigma \operatorname{ad}(h) X_{i}$ are regular elements which commute, so we must have $\sigma \operatorname{ad}(h) \mathfrak{h}_{i}=\operatorname{ad}(h) \mathfrak{h}_{i}$. This means $[\operatorname{ad}(h) X, \sigma \operatorname{ad}(h) X]=0$, so $h^{-1} x \in \operatorname{Crit}(g)$. Thus we have Crit $(g)=$ $\cup$ Crit $\left(\exp X_{i}\right)$. 
We assume now that $g=\exp X$ with $X$ a regular element of $G$, and $x=K$ is in Crit $(g)$.

If $\mathfrak{h}$ is the Cartan algebra of $G$ containing $X$, then the assumption that $x$ is a critical point implies $[X, \sigma X]=0$ which means $\sigma \mathfrak{h}=\mathfrak{h}$. Suppose $h \in \exp m$ is such that $h^{-1} x \in \operatorname{Crit}(g)$. Then $[\operatorname{ad}(h) X, \sigma \operatorname{ad}(h) X]=0$, or equivalently, $\operatorname{ad}(h) \mathfrak{h}$ $=\sigma \operatorname{ad}(h) \mathfrak{h}$. But this just means $\operatorname{ad}\left(h^{2}\right) \mathfrak{h}=\sigma \mathfrak{h}=\mathfrak{h}$, so $h^{2} \in$ normalizer of $\mathfrak{h}$ in $G$. If $h$ is sufficiently close to the identity $e$, then this condition implies $h \in T$, where $T$ is the identity component of the normalizer. $T$ is the maximal torus of $G$ corresponding to $\mathfrak{h}$, and equals $Z_{G}^{0}(g)=$ identity component of the centralizer of $g$ in $G$. If $y$ is in the same component of Crit $(g)$ as $x$, then we cover a curve $c$ in Crit $(g)$ from $x$ to $y$ by neighborhoods $U_{j}$ where $U_{j}=V x_{j}$ for a neighborhood $V \subset T$ of $e$, and a finite number of points $x_{i} \in c$ such that $x_{0}=x$, $x_{n}=y$, and $x_{j} \in V x_{j-1}$ for all $1 \leq j \leq n$. Since $c$ is compact this is possible for some $n$. We choose $n$ large enough and $V$ so small that the transvection $h \in(\exp m) \cap V$ always satisfies the property that if $h^{2} \in$ normalizer of $T$ then $h \in T$. Note that the set $m$ of transvections may change with $j$, but this does not affect the above construction. Then $x_{j}=g_{j} x_{j-1}$ for $g_{j} \in V$, so $y=g_{n} g_{n-1}$ $\cdots g_{1} x$, which means $y \in T x$. This shows that the component of Crit $(g)$ which contains $x$ is contained in $T x$. The other inclusion is obvious since $T$ is in the centralizer of $g$. Thus Crit $(g)=\bigcup_{m} T x_{m}$ for a set $\left\{x_{m}\right\}$ of representative elements of the components of Crit $(g)$.

If $g$ is not regular then $Z_{G}^{0}(g)=\bigcup_{i} T_{i}$, where the $T_{i}$ are the distinct maximal tori containing $g$. Therefore

$$
\begin{aligned}
\operatorname{Crit}(g) & =\bigcup_{i} \operatorname{Crit}\left(\exp X_{i}\right)=\bigcup_{i} \bigcup_{m_{i}} T_{i} x_{m_{i}} \\
& \subseteq\left(\bigcup_{i} T_{i}\right) \cdot\left(\bigcup_{m} x_{m}\right)=\bigcup_{m} Z_{G}^{0}(g) \cdot x_{m},
\end{aligned}
$$

where $\left\{x_{m}\right\}$ is a set of representatives of the components of Crit $(g)$. Since $\bigcup_{m} Z_{G}^{0}(g) x_{m} \subset$ Crit $(g)$, the result follows.

(2.3.3) Remark. In the case where $X$ is a regular element we see from the proof of the above theorem that in fact the orbit of $x$ by the normalizer of $\mathfrak{h}$ in $G$ is contained in Crit $(\exp X)$. It would be interesting to know if this is an equality.

(2.3.4) Corollary. If $g=\exp X$ for a regular element $X$ of $G$, then $C$ rit $(g)$ is a flat totally geodesic submanifold of $M$.

Proof. Since the components of Crit ( $g$ ) are orbits by an abelian subgroup, they must be flat, and are totally geodesic because this subgroup is invariant by the symmetry $\sigma$ of $G$ corresponding to the geodesic symmetry at each point of Crit $(g)$.

(2.3.5) Example. In the proof Corollary 2.3 .4 we use regularity of $X$ to 
get that $Z_{G}^{0}(\exp X)$ is invariant by $\sigma$. This in turn requires $Z_{G}(X)$ to be $\sigma$-invariant. The assumption of regularity cannot be dropped, as seen from the following example of J.A. Wolf : Let $M=S U(6) / S O(6)$, and let $e_{1}, \cdots, e_{6}$ be a basis of $S u(6)$. Let $X_{K}$ and $X_{m}$ have eigenvalues $\sqrt{-1},-\sqrt{-1}, 2 \sqrt{-1}$, $-2 \sqrt{-1}, 10^{20} \sqrt{-1},-10^{20} \sqrt{-1}$ and $\sqrt{-1}, \sqrt{-1}, 2 \sqrt{-1}, 2 \sqrt{-1},-3 \sqrt{-1}$, $-3 \sqrt{-1}$, respectively, corresponding to the vectors $e_{1}, \cdots, e_{6}$. Then $\left[X_{K}, X_{m}\right]$ $=0$. $X_{K}+X_{m}$ has eigenvalues $0,2 \sqrt{-1}, 4 \sqrt{-1},-3 \sqrt{-1}+10^{20} \sqrt{-1}$, $-3 \sqrt{-1}-10^{20} \sqrt{-1}$ corresponding to the eigenspaces spanned by $\left\{e_{2}, e_{4}\right\}$, $\left\{e_{1}\right\},\left\{e_{3}\right\},\left\{e_{5}\right\},\left\{e_{6}\right\}$ respectively, and $X_{K}-X_{m}$ has eigenvalues $0,-2 \sqrt{-1}$, $-4 \sqrt{-1}, 3 \sqrt{-1}+10^{20} \sqrt{-1}, 3 \sqrt{-1}-10^{20} \sqrt{-1}$, corresponding to the eigenspaces spanned by $\left\{e_{1}, e_{3}\right\},\left\{e_{2}\right\},\left\{e_{4}\right\},\left\{e_{5}\right\},\left\{e_{6}\right\}$ respectively. The centralizers of $X_{\boldsymbol{K}}+X_{m}$ and $\boldsymbol{X}_{\boldsymbol{K}}-X_{m}$ consist of matrices which are scalar multiples of the identity in each of their eigenspaces; but as the eigenspaces do not correspond, the centralizers are not equal. If $X=X_{K}+X_{m}$ then $\sigma X=X_{K}-X_{m}$; and clearly $Z_{G}(\sigma X)=\sigma Z_{G}(X)$, so we have $\sigma Z_{G}(X) \neq Z_{G}(X)$.

(2.4) In this section wo consider symmetric spaces of noncompact type.

(2.4.1) Theorem. Let $M=G / K$ be a connected Riemannian symmetric space of non-compact type, and assume $g \in G$ is any isometry. If $x \in \mathrm{Crit}(\mathrm{g})$, then Crit $(g)=Z_{G}^{0}(g) \cdot x$.

Proof. Since $M$ is simply connected with curvature $K \leq 0$ there are no cut points so every isometry is of small displacement, and every pair of points is joined by a unique minimizing geodesic.

Suppose $y \neq x$ is another critical point, and let $(\exp s S) x, S \in m$, be the geodesic from $x$ to $y$. We assume $S$ is transverse to the geodesic $c$ from $x$ to $g x$. Construct the surface $Q$ as in Chapter I, and let $T \in m$ be the tangent to $c$. Then by Theorem 1.3.6 we have that $Q$ is flat and the vector fields $S$ and $T$ are parallel on $Q$, where flatness implies $[S, T]=0$. Now in a symmetric space $d L_{\text {expss }}(T)$ is parallel along $(\exp s S) x$, and since $T$ itself is parallel, $T=$ $d L_{\exp s S}(T)$. Therefore the translation $L_{\exp s s}$ takes the geodesic $(\exp t T) x$ to the geodesic from $(\exp s S) x$ to $g(\exp s S) x$ for each $s$. Thus $(\exp s S) g x=g(\exp s S) x$, or $g^{-1}(\exp -s S) g(\exp s S) x=x$ which means $g^{-1}(\exp -s S) g(\exp s S) \in K$. Now $g=(\exp T) k$ with $T \in m, k \in K$, and $[S, T]=0$. Therefore we get $k^{-1}(\exp -T)$ $\cdot(\exp -s S)(\exp T) k(\exp s S) \in K$, which implies $(\exp -s S) k(\exp s S) \in K$ for all $s$. Then $d R_{k}(S)-d L_{k}(S) \in K$, or $S-\operatorname{ad}(k) S \in K$.

Since $S \in m$ and $k \in K, \operatorname{ad}(k) S \in m$, so $\operatorname{ad}(k) S=S$. Thus $g(\exp s S) g^{-1}=$ $(\exp T) k(\exp s) k^{-1}(\exp -T)=(\exp T)(\exp s \operatorname{ad}(k) S)(\exp -T)=\exp s \operatorname{ad}(\exp T) S$ $=\exp s S$ for every $s$. Thus Crit $(g) \subset Z_{G}^{0}(g) x$. The other inclusion is obvious, so Crit $(g)=Z_{G}^{0}(g) x$.

(2.5) In [7] and [8] R. Hermann discussed the critical points of the squared length function $f_{x}$ of a Killing vector field $X$. We shall reformulate a part of Theorem 1 in [7], and then a comparison with our results show that in the case of a symmetric space, the critical manifold of $f_{X}$ coincides with that of $g_{\iota}=$ 
$\exp t X$ for any small $t$. That Crit $\left(\exp t_{1} X\right)=$ Crit $\left(\exp t_{2} X\right)$ for any small $t_{1}, t_{2}$ is obvious from Theorem 2.2.2 for the case of compact spaces.

We again fix a decomposition $\boldsymbol{G}=\boldsymbol{K}+\boldsymbol{m}$ of the Lie algebra $\boldsymbol{G}$ of $\boldsymbol{G}$, where $M=G / K$ is the symmetric space and $K($ resp. $m)$ the +1 (resp. -1$)$ eigenspaces of the symmetry $\sigma$. For each $g \in G$, we let $P_{g}: G \rightarrow \operatorname{ad}(g) m$ be the projection. Notice that $P_{g}$ depends only on $g K$.

It is easy to see that $\operatorname{ad}(g) \circ P_{e}=P_{g} \circ \operatorname{ad}(g)$, so $P_{g}=\operatorname{ad}(g) \circ P_{e} \circ \operatorname{ad}\left(g^{-1}\right)$. Now for every $X \in G$ we have a Killing vector field, which we also denote by $X$, on $M$ coming from the 1-parameter group $\exp t X$ of isometries of $M$. Identify the tangent space of $M$ at $g K$ with $\operatorname{ad}(g) m$ for each $g \in G$; then we may view $P_{0} X$ as a vector field on $M$. In fact, $P_{\theta} X$ is the Killing vector field of the 1-parameter group $\exp t X$. Let $\langle$,$\rangle be the invariant metric on M$, so that

$$
\begin{aligned}
f_{X}(g X) & =\left\langle P_{g} X, P_{g} X\right\rangle=\left\langle\operatorname{ad}(g) \circ P_{e} \circ \operatorname{ad}\left(g^{-1}\right) X, \operatorname{ad}(g) P_{e} \operatorname{ad}\left(g^{-1}\right) X\right\rangle \\
& =\left\langle P_{e} \circ \operatorname{ad}\left(g^{-1}\right) X, P_{e} \circ \operatorname{ad}\left(g^{-1}\right) X\right\rangle .
\end{aligned}
$$

$f_{X}$ is evidently differentiable on $M$. We will use the abbreviation $f_{X}(g K)=$ $f_{X}(g)$. Then $f_{X}$ has a critical point at $g K$ exactly when

$$
\left.\frac{d}{d t}\right|_{t=0} f_{X}((\exp t H) g)=0
$$

for all $H \in m$. Now

$$
\begin{aligned}
f_{X}((\exp t H) g) & =\left\langle P_{e} \circ \operatorname{ad}((\exp t H) g) X, P_{e} \circ \operatorname{ad}((\exp t H) g) X\right\rangle \\
& =\left\langle\left(e^{t \operatorname{ad} H} \circ \operatorname{ad}(g) X\right)_{m},\left(e^{t \operatorname{ad} I I} \circ \operatorname{ad}(g) X\right)_{m}\right\rangle .
\end{aligned}
$$

Here $e^{t \mathrm{ad} H}=\cosh (t \operatorname{ad} H)+\sinh (t \operatorname{ad} H), \cosh$ and sinh denoting the usual power series.

Since $H \in m$, and $M$ is a symmetric space, we have $\cosh (t \operatorname{ad} H) m \subset m$, $\cosh (t \operatorname{ad} H) K \subset K, \sinh (t \operatorname{ad} H) m \subset K$, and $\sinh (t \operatorname{ad} H) K \subset m$, so

$$
\begin{aligned}
\left(e^{t \operatorname{ad} H}\right. & \circ \operatorname{ad}(g) X)_{m} \\
& =\left\{[\cosh (t \operatorname{ad} H)+\sinh (t \operatorname{ad} H)]\left[(\operatorname{ad}(g) X)_{m}+(\operatorname{ad}(g) X)_{K}\right]\right\}_{m} \\
& =\cosh (t \operatorname{ad} H)(\operatorname{ad}(g) X)_{m}+\sinh (t \operatorname{ad} H)(\operatorname{ad}(g) X)_{K} .
\end{aligned}
$$

Thus

$$
\left.\frac{d}{d t}\right|_{t=0}\left(e^{t \operatorname{ad} I I} \operatorname{ad}(g) X\right)_{m}=\operatorname{ad} H(\operatorname{ad}(g) X)_{K} .
$$

Now 


$$
\begin{aligned}
\left.\frac{d}{d t}\right|_{t=0} & f f_{X}((\exp t H) g) \\
= & 2\left\langle\left.\frac{d}{d t}\right|_{t=0}\left(e^{t \operatorname{ad} I I} \operatorname{ad}(g) X\right)_{m},(\operatorname{ad}(g) X)_{m}\right\rangle \\
= & 2\left\langle\operatorname{ad} H(\operatorname{ad}(g) X)_{K},(\operatorname{ad}(g) X)_{m}\right\rangle \\
= & 2\left\langle H,\left[(\operatorname{ad}(g) X)_{K},(\operatorname{ad}(g) X)_{m}\right]\right\rangle .
\end{aligned}
$$

Now $\langle$,$\rangle is non-degenerate so the above vanishes for all H \in m$ if and only if $\left[(\operatorname{ad}(g) X)_{K},(\operatorname{ad}(g) X)_{m}\right]=0$. Since we are in a symmetric space this is equivalent to $[\operatorname{ad}(g) X, \sigma \operatorname{ad}(g) X]=0$.

We assume that $M$ is complete and connected so that every point $g \cdot K$ in $M$ can be represented by a transvection; that is, an element $g \in G$ such that $\sigma g=$ $g^{-1}$. Every $g \in G$ can be expressed as a product $g=p k$ with $k \in K$ and $\sigma p=$ $p^{-1}$, so that $\operatorname{ad}(g) m=\operatorname{ad}(p) m$, which shows that $P_{g}$ depends only on the transvective component $p$ of $g$, so we may assume $g$ is a transvection. Then

$$
\begin{aligned}
{[\operatorname{ad}(g) X, \sigma \operatorname{ad}(g) X] } & =\left[\operatorname{ad}(g) X, \operatorname{ad}\left(g^{-1}\right) \sigma X\right] \\
& =\operatorname{ad}\left(g^{-1}\right)\left[\operatorname{ad}\left(g^{2}\right) X, \sigma X\right]
\end{aligned}
$$

Thus $g^{-1} K$ is a critical point of $f_{X}$ if and only if $\left[\operatorname{ad}\left(g^{2}\right) X, \sigma X\right]=0$. This is the first part of Theorem 1 in [7].

Now let $M$ be connected, symmetric and of non-compact type, and consider the critical set Crit $\left(f_{X}\right)$ of $f_{X}$.

(2.5.1) Theorem.

$$
\text { Crit }\left(f_{X}\right)=\text { Crit }(\exp X)=Z_{G}^{0}(\exp X) \cdot x
$$

for any $x \in C$ Crit $(\exp X)$ if $X$ is sufficiently small.

Proof. By the remarks of (2.5), we have that for $h \in \exp m, h K$ is a critical point of $f_{X}$ if and only if $\left[\operatorname{ad}\left(h^{-2}\right) X, \sigma X\right]=0$. We will find the tangent space of the critical set of $f_{X}$ at $x=K$ assuming $x$ is a critical point of $f_{X}$. Suppose $H(t)$ is a $C^{\infty}$-curve in $m$ with $H(0)=0$ such that $\exp H(t) x \in$ Crit $\left(f_{X}\right)$ for small $t$. Then $[\operatorname{ad}(\exp -2 H(t)) X, \sigma X]=0$ for all $t$ near zero. Assume that

$$
\left.\frac{d}{d t}\right|_{t=0} H(t)=V
$$

Now $\operatorname{ad} \sigma X\left(e^{-2 \text { ad } H(t)} X\right)=0$ so

$$
\operatorname{ad} \sigma X\left(X-2 \operatorname{ad} H(t)(X)+4(\operatorname{ad} H(t))^{2} X-\cdots\right)=0 .
$$

Differentiating at $t=0$, this shows that $2 \operatorname{ad} \sigma X \operatorname{ad} V(X)=0$, that is, 


$$
\begin{aligned}
0=[S-T,[V, S+T]]=[S,[V, S]]-[T,[V, S]] \\
+[S,[V, T]]-[T,[V, T]] \\
=(\operatorname{ad} T)^{2} V-(\operatorname{ad} S)^{2} V+(\operatorname{ad} T \operatorname{ad} S-\operatorname{ad} S \operatorname{ad} T) V .
\end{aligned}
$$

Here $X=S+T$ with $S \in K$ and $T \in m$. Now $(\operatorname{ad} T)^{2} V$ and $(\operatorname{ad} S)^{2} V$ are in $m$, and ad $T$ ad $S(V)$ and ad $S$ ad $T(V)$ are in $K$, so in particular we get $(\operatorname{ad} T)^{2} V-$ $(\operatorname{ad} S)^{2} V=0$. The Killing form $B$ is negative definite on $K$ and positive definite on $m$, so we can define a new form $B_{\sigma}$ on $G$ by $B_{\sigma}(X, Y)=-B(X, \sigma Y)$. $B_{0}$ is positive definite on $G$, but no longer invariant under the adjoint action of $G$ on $\boldsymbol{G}$. Let $Y, Z \in G$. Then

$$
\begin{aligned}
B_{o}(\operatorname{ad} S(Y), Z) & =-B(\operatorname{ad} S(Y), \sigma Z)=B(Y, \operatorname{ad} S(\sigma Z)) \\
& =B(Y, \sigma(\operatorname{ad} S(Z)))=-B_{o}(Y, \operatorname{ad} S(Z)),
\end{aligned}
$$

so ad $S$ is skew-symmetric with respect to $B_{\sigma}$. Similarly,

$$
\begin{aligned}
B_{\sigma}(\operatorname{ad} T(Y), Z) & =-B(\operatorname{ad} T(Y), \sigma Z)=B(Y, \operatorname{ad} T(\sigma Z)) \\
& =-B(Y, \sigma(\operatorname{ad} T(X)))=B_{\diamond}(Y, \operatorname{ad} T(Z)),
\end{aligned}
$$

so ad $T$ is symmetric on $G$ with respect to $B_{\sigma}$. Since $[X, \sigma X]=0$, we have $[S, T]=0$ so ad $S$ and ad $T$ commute on $G$. Now ad $S$ has pure imaginary eigenvalues since it is skew, and ad $T$ has real eigenvalues since it is symmetric. Therefore $(\operatorname{ad} S)^{2}$ is negative-semidefinite, and $(\operatorname{ad} T)^{2}$ is positive semi-definite. This means that if $(\operatorname{ad} S)^{2} V=(\operatorname{ad} T)^{2} V$, we must have $(\operatorname{ad} S)^{2} V=0=(\operatorname{ad} T)^{2} V$. $0=B_{\sigma}\left((\operatorname{ad} S)^{2} V, V\right)=\left(B_{a}(\operatorname{ad} S(V), \operatorname{ad} S(V))\right.$ and $0=B_{\sigma}\left((\operatorname{ad} T)^{2} V, V\right)=$ $B_{\sigma}(\operatorname{ad} T(V)$, ad $T(V))$ so ad $S(V)=0=\operatorname{ad} T(V)$ since $B_{\sigma}$ is positive definite. Hence $[V, X]=0$. Since $[V, X]=0$ implies $(\exp t V) x \in$ Crit $\left(f_{X}\right)$ for all $t$, we see that the tangent space of Crit $\left(f_{X}\right)$ at $x$ is $Z_{m}(X)=$ centralizer of $X$ in $m$. In Theorem $3.1(f)$ of [8] it is shown that Crit $\left(f_{X}\right)$ is connected and convex so that every point $y \in$ Crit $\left(f_{X}\right)$ lies on a geodesic in Crit $\left(f_{X}\right)$ which passes through $x$; this geodesic has the form $(\exp t H) x$ for $H \in m$, and the above shows $H \in Z_{G}(X)$. Thus Crit $\left(f_{X}\right)=Z_{G}^{0}(\exp X) \cdot x$. Now fix $x \in \operatorname{Crit}\left(f_{X}\right)$. If we let $x=$ $K$, then we have $[X, \sigma X]=0$ so $X=S+T$ with $S \in K, T \in m$ and $[S \cdot T]=0$. Therefore $\operatorname{ad}(\exp S) T=T$ and $x \in$ Crit $(\exp t X)$ for all sufficiently small $t$. Conversely, suppose $x \in \mathrm{Crit}(\exp t X)$ for $t$ small enough so that $\exp t X=$ $(\exp T)(\exp S)$ for unique stortest $T \in m, s \in K$ and such that $(\exp T)(\exp S)=$ $(\exp S)(\exp T)$ if and only if $[S, T]=0$. It is possible to choose $t$ so small by an argument used in the proof of Lemma 2.2.1. The choice of how small $t$ has to be depends on $x$, and since $M$ is non-compact there may be no value which works for all $x$. However, the above shows that this particular $x$ is in Crit $\left(f_{X}\right)$. But since Crit $\left(f_{X}\right)=Z_{G}^{0}(\exp t X) \cdot x$ and Crit $(\exp t X)=Z_{G}^{0}(\exp t X) \cdot x$, we have Crit $\left(f_{X}\right)=$ Crit $(\exp t X)$.

We now compute the Hessian $\mathscr{H}_{X}$ of $f_{X}$ at $\mathrm{g}=e$. To do this, let $H_{1}, H_{2} \in m$ 
and differentiate the expression $f_{X}\left(\left(\exp s H_{1}\right)\left(\exp t H_{2}\right) e\right)$ at $t=\mathrm{s}=0$.

$$
\begin{aligned}
\left.\frac{\partial^{2}}{\partial s \partial t}\right|_{0,0} & f_{X}\left(\left(\exp s H_{1}\right)\left(\exp t H_{2}\right)\right) \\
= & \left.\frac{\partial^{2}}{\partial s \partial t}\right|_{0,0}\left\langle\left(e^{s \mathrm{ad} H_{1}} e^{t \mathrm{ad} H_{2}} X\right)_{m},\left(e^{s \mathrm{ad} H_{1}} e^{t \text { ad } H_{2}} X\right)_{m}\right\rangle \\
= & \left.2 \frac{\partial}{\partial s}\right|_{0}\left\langle\left.\frac{\partial}{\partial t}\right|_{0}\left(e^{s \mathrm{ad} H_{1}} e^{t \text { ad } H_{2}} X\right)_{m l},\left(e^{s \mathrm{ad} H_{1}} e^{t \text { ad } I_{2}} X\right)_{m}\right\rangle \\
= & 2\left\{\left\langle\left.\frac{\partial^{2}}{\partial s \partial t}\right|_{0,0}\left(e^{s \mathrm{ad} I_{1}} e^{t \text { ad } H_{2}} X\right)_{m}, X_{m}\right\rangle\right. \\
& \quad+\left\langle\left.\frac{\partial}{\partial t}\right|_{0,0}\left(e^{s \mathrm{ad} I_{1}} e^{t \text { ad } H_{2}} X\right)_{m},\left.\frac{\partial}{\partial s}\right|_{0,0}\left(e^{s \mathrm{ad} H_{1}} e^{\left.\ell \text { ad } I_{2} X\right)_{m}}\right\rangle\right\} .
\end{aligned}
$$

Now $e^{t \text { ad } I I}=\cosh (t \operatorname{ad} H)+\sinh (t \operatorname{ad} H)$, and

$$
\left.\frac{d}{d t}\right|_{0} \cosh (t \operatorname{ad} H)=0,\left.\frac{d}{d t}\right|_{0} \sinh (t \operatorname{ad} H)=\operatorname{ad} H ;
$$

also,

$$
\begin{aligned}
\left(e^{s \text { ad } H_{1}} e^{t \text { ad } H_{2}} X\right)_{m} \\
=\left\{\left(\cosh \left(s \operatorname{ad} H_{1}\right)+\sinh \left(s \operatorname{ad} H_{1}\right)\right)\left(\cosh \left(t \operatorname{ad} H_{2}\right)+\sinh \left(t \operatorname{ad} H_{2}\right)\right) X\right\}_{m} \\
=\cosh \left(s \operatorname{ad} H_{1}\right) \cosh \left(t \operatorname{ad} H_{2}\right) X_{m}+\cosh \left(s \operatorname{ad} H_{1}\right) \sinh \left(t \operatorname{ad} H_{2}\right) X_{K} \\
\\
\quad+\sinh \left(s \operatorname{ad} H_{1}\right) \cosh \left(t \operatorname{ad} H_{2}\right) X_{K}+\sinh \left(s \operatorname{ad} H_{1}\right) \sinh \left(t \operatorname{ad} H_{2}\right) X_{m} .
\end{aligned}
$$

So,

$$
\left.\frac{\partial^{2}}{\partial s \partial t}\right|_{0,0}\left(e^{s \operatorname{ad} I / 1} e^{t \operatorname{ad} / I_{2}} X\right)_{m}=\operatorname{ad} H_{1} \operatorname{ad} H_{2}\left(X_{m}\right)
$$

and

$$
\begin{aligned}
& \left.\frac{\partial}{\partial t}\right|_{0,0}\left(e^{s \text { ad } H_{1}} e^{t \text { ad } I_{2}} X\right)_{m}=\operatorname{ad} H_{2}\left(X_{K}\right), \\
& \left.\frac{\partial}{\partial s}\right|_{0,1}\left(e^{s \text { ad } I_{1}} e^{\ell \text { ad } I_{2}} X\right)_{m}=\operatorname{ad} H_{1}\left(X_{K}\right) .
\end{aligned}
$$

Thus,

$$
\begin{aligned}
& \left.\frac{\partial^{2}}{\partial s \partial t}\right|_{0,0} f_{X}\left(\left(\exp s H_{1}\right)\left(\exp t H_{2}\right)\right) \\
& \quad=2\left(\left\langle\operatorname{ad} H_{1} \operatorname{ad} H_{2}\left(X_{m}\right), X_{m}\right\rangle+\left\langle\operatorname{ad} H_{2}\left(X_{K}\right), \operatorname{ad} H_{1}\left(X_{K}\right)\right\rangle\right) .
\end{aligned}
$$


For any $Z \in G, \operatorname{ad} Z$ is skew-symmetric with respect to $\langle$,$\rangle so the above be-$ comes:

$$
\begin{gathered}
2\left(\left\langle\operatorname{ad} X_{K}\left(H_{2}\right), \operatorname{ad} X_{K}\left(H_{1}\right)\right\rangle-\left\langle\operatorname{ad} X_{m}\left(H_{2}\right), \operatorname{ad} X_{m}\left(H_{1}\right)\right\rangle\right) \\
=2\left(\left\langle\left(\operatorname{ad} X_{m}\right)^{2} H_{2}, H_{1}\right\rangle-\left\langle\left(\operatorname{ad} X_{K}\right)^{2} H_{2}, H_{1}\right\rangle\right) \\
=2\left\langle\left(\left(\operatorname{ad} X_{m}\right)^{2}-\left(\operatorname{ad} X_{K}\right)^{2}\right) H_{2}, H_{1}\right\rangle .
\end{gathered}
$$

It is clear that $\left(\operatorname{ad} X_{m}\right)^{2}-\left(\operatorname{ad} X_{K}\right)^{2}$ is symmetric with respect to $\langle$,$\rangle . Thus$ we have

\section{(2.5.2) Theorem.}

$$
\begin{aligned}
\mathscr{H}_{X}\left(H_{1}, H_{2}\right) & =\left(\text { Hessian of } f_{X}\right)\left(H_{1} H_{2}\right) \\
& =2\left\langle\left(\left(\operatorname{ad} X_{m}\right)^{2}-\left(\operatorname{ad} X_{K}\right)^{2}\right) H_{1}, H_{2}\right\rangle .
\end{aligned}
$$

(2.5.3) Corollary. If $M$ is of non-compact type, then Crit $\left(f_{X}\right)$ is a nondegenerate critical manifold. If $M$ is of compact type and $X$ is a regular element, then $\mathrm{Crit}\left(f_{X}\right)$ is also non-degenerate.

Proof. The nullity of $\mathscr{H}_{X}$ is the nullity of the form $\left(\operatorname{ad} X_{m}\right)^{2}-\left(\operatorname{ad} X_{K}\right)^{2}$. The proof of Theorem 2.5.1 shows that this is just $Z_{m}(X)=$ (centralizer of $X$ in $m)=\left(\right.$ tangent space of Crit $\left(f_{X}\right)$ at $\left.x=K\right)$, if $M$ is non-compact.

Now assume $M$ is of compact type and $X \in B_{r}$, so that $\operatorname{Crit}\left(f_{X}\right)=\operatorname{Crit}(\exp X)$. We assume $X$ is a regular element of $G$, so $Z_{G}(X)=$ Cartan algebra containing $X$. Since we assume $x=K$ is in Crit $\left(f_{X}\right)$, we have $[X, \sigma X]=0$, so $Z_{G}(X)$ $=Z_{G}(\sigma X)$. Now $H \in$ nullity of $\mathscr{H}_{X}$ if and only if $\operatorname{ad} X \operatorname{ad} \sigma X(H)=0$; equivalently, $\operatorname{ad} \sigma X(H) \in Z_{G}(X)=Z_{G}(\sigma X)$, or $(\operatorname{ad}(\sigma X))^{2} H=0$. Similarly, $(\operatorname{ad} X)^{2} H=0$. Now

$$
(\operatorname{ad} \sigma X)^{2} H=\left(\operatorname{ad} X_{K}\right)^{2} H+\left(\operatorname{ad} X_{m}\right)^{2} H-2 \operatorname{ad} X_{K} \operatorname{ad} X_{m}(H),
$$

and

$$
(\operatorname{ad} X)^{2} H=\left(\operatorname{ad} X_{K}\right)^{2} H+\left(\operatorname{ad} X_{m}\right)^{2} H+2 \operatorname{ad} X_{K} \text { ad } X_{m}(H) .
$$

Therefore, $\left(\operatorname{ad} X_{K}\right)^{2} H+\left(\operatorname{ad} X_{m}\right)^{2} H=0$. Since also $\left(\operatorname{ad} X_{K}\right)^{2} H=\left(\operatorname{ad} X_{m}\right)^{2} H$, we have $\left(\operatorname{ad} X_{K}\right)^{2} H=0$ and $\left(\operatorname{ad} X_{m}\right)^{2} H=0$, which implies that $\operatorname{ad} X_{K}(H)=0$ and ad $X_{m}(H)=0$, so $H \in Z_{G}(X)$. Now every $H \in Z_{G}(X)$ is in the nullity of $\mathscr{H}_{X}$, so Crit $\left(f_{X}\right)$, is non-degenerate.

(2.6) We now treat the Euclidean space $\boldsymbol{R}^{n}$. Let $E(n)$ be the Euclidean group of isometries of $\boldsymbol{R}^{n}$; then each $g \in E(n)$ is a pair $g=(A, v)$ for $A \in 0(n)$, $v \in \boldsymbol{R}^{n}$, and acts on $\boldsymbol{R}^{n}$ as follows: if $x \in \boldsymbol{R}^{n}$ then $g x=A x+v . E(n)$ is a semidirect product of $O(n)$ with $\boldsymbol{R}^{n}$, so that $\boldsymbol{R}^{n}=E(n) / 0(n)$ is a Riemannian homogeneous space with normal metric. Furthermore, if $A \in O(n), v \in \boldsymbol{R}^{n}$ then $\operatorname{ad}(A) v$ $=A v$. We now choose a particular isometry $g=(A, v)$ and find Crit $(g)$. Note 
that since $R^{n}$ has no cut points we can use Corollary 2.1.2 for any $g \in E(n)$. Assume that Crit $(g) \neq \emptyset$, and choose $x \in \mathrm{Crit}(g)$ to be the origin of $\boldsymbol{R}^{n}$. Then we must have $v=\operatorname{ad}(A) v=A v$. Now let $h \in R^{n}$ be any vector. Then

$$
\begin{aligned}
h \cdot g \cdot h^{-1}(y) & =h+g(y-h) \\
& =h+v+A(y-h)=h+v-A h+A y,
\end{aligned}
$$

so that $h \cdot g \cdot h^{-1}=(A, h+v-A h)$. Now $-h \in$ Crit $(g)$ if and only if $h+v$ $-A h=A(h+v-A h)$, that is, $h-2 A h+A^{2} h=0$, which means $(I-A)^{2} h=0$.

Since $A \in O(n), A=\{A_{1}, \cdots, A_{k}, \underbrace{1, \cdots, 1}_{p},-1, \cdots,-1\}$ with

$$
A_{i}=\left(\begin{array}{rr}
\cos \theta_{i} & \sin \theta_{i} \\
-\sin \theta_{i} & \cos \theta_{i}
\end{array}\right), \quad \text { and } \quad \theta_{i} \neq n \pi \text {. }
$$

Then

$$
(A-I)^{2}=\left\{\left(A_{1}-I\right)^{2}, \cdots,\left(A_{k}-I\right)^{2}, 0, \cdots, 0,4, \cdots, 4\right\} .
$$

If $(A-I)^{2} h=0$ for $h=\left(h_{1}, \cdots, h_{n}\right)$, we must have

$$
\left(A_{i}-I\right)^{2}\left(\begin{array}{l}
h_{2 i-1} \\
h_{2 i}
\end{array}\right)=0 \quad \text { for } i=1, \cdots, k,
$$

and $h_{2 k+p+j}=0$ for $j=1, \cdots, n-2 k-p$. Now $\operatorname{det}\left(A_{i}-I\right)^{2}=\left(\operatorname{det}\left(A_{i}-I\right)\right)^{2}$ $=\left(\left(\cos \theta_{i}-1\right)^{2}+\sin ^{2} \theta_{i}\right)^{2}$, and this is zero only when $\theta_{i}=n \pi$ which is impossible. Therefore, $h_{j}=0$ for $j=1, \cdots, 2 k$, and we have $A h=h$. Conversely, $A h=h$ clearly implies $A(h+v-A h)=h+v-A h$, so Crit $(g)=$ $Z_{E(n)}(g) \cdot x$. Hence we have proved

(2.6.1) Theorem. Let $M$ be a Euclidean space and $g: M \rightarrow M$ any isometry. If $x \in$ Crit $(g)$, then Crit $(g)=Z_{E(n)}(g) \cdot x$.

(2.7) Suppose $M=M^{\prime} \times M^{\prime \prime}$ is the Riemannian product of Rimannian manifolds $M^{\prime}$ and $M^{\prime \prime}$, and let $g$ be the product metric on $M$. Suppose $f: M$ $\rightarrow M$ is an isometry of small displacement satisfying $f=f^{\prime} \times f^{\prime \prime}$, where $f^{\prime}: M^{\prime}$ $\rightarrow M^{\prime}$ and $f^{\prime \prime}: M^{\prime \prime} \rightarrow M^{\prime \prime}$ are isometries, and let $b(s)$ be a curve through some point $x=\left(x^{\prime}, x^{\prime \prime}\right) \in M$. Then $\delta_{f}(b(s))=\int_{0}^{o} \sqrt{ } g(T, T) d t$, where $T$ is as defined in (1.2).

$$
\begin{aligned}
\frac{d}{d s} \delta_{f}(b(s)) & =\int_{0}^{a} \frac{\partial}{\partial s} \sqrt{g\left(T^{\prime}, T^{\prime}\right)+g\left(T^{\prime \prime}, T^{\prime \prime}\right)} d t \\
& =\int_{0}^{a} \frac{g\left(\nabla_{X}, T^{\prime}, T^{\prime}\right)+g\left(\nabla_{X^{\prime}} T^{\prime \prime}, T^{\prime \prime}\right)}{\sqrt{g(T, T)}} d t
\end{aligned}
$$

where $T^{\prime}, X^{\prime}$ and $T^{\prime \prime}, X^{\prime \prime}$ are the components of $Q^{*} \partial / \partial t, Q_{*} \partial / \partial s$ in $T\left(M^{\prime}\right)$ 
and $T\left(M^{\prime \prime}\right)$ respectively. From this it is clear that the derivative vanishes at $x$ for all values of $X^{\prime}+X^{\prime \prime}$ exactly when $x^{\prime} \in$ Crit $\left(f^{\prime}\right)$ and $x^{\prime \prime} \in \operatorname{Crit}\left(f^{\prime \prime}\right)$.

(2.7.1) Theorem. Let $M$ be a simply connected Riemannian symmetric space with $M=M_{0} \times M_{1} \times \cdots \times M_{p}$, where $M_{0}$ is a Euclidean space and the $M_{i}, 1 \leq i \leq k$, are irreducible. Suppose $g \in I^{0}(M)=I^{0}\left(M_{0}\right) \times I^{0}\left(M_{1}\right) \times \cdots \times$ $I^{0}\left(M_{k}\right)$, and the components $g_{i}$ of $g$ acting on the $M_{i}$ which are compact satisfy the hypotheses of Theorem 2.3.2. If $x \in \mathrm{Crit}(\mathrm{g})$, then the component of Crit $(\mathrm{g})$ containing $x$ is $Z_{I^{0}(M)}^{0}(g) \cdot x$.

Proof. From the above remarks we see that Crit $(g)=\operatorname{Crit}\left(g_{0}\right) \times \operatorname{Crit}\left(g_{1}\right)$ $\times \cdots \times$ Crit $\left(g_{k}\right)$. Then the result follows from Theorems 2.3.2, 2.4.1, and 2.5.1.

(2.7.2) Lemma. Let $\bar{M} \stackrel{\pi}{\longrightarrow} M$ be a Riemannian covering of Riemannian manifolds with simply connected $\bar{M}$, and $f: M \rightarrow M$ an isometry of small displacement. Then there is a unique lift $\bar{f}: \bar{M} \rightarrow \bar{M}$ of $f$ which is an isometry covering $f$, and such that $\rho(x, f(x))=\tilde{\rho}(\tilde{x}, \tilde{f}(\tilde{x}))$ for all $\tilde{x}$ such that $\pi(\tilde{x})=x$, $x \in M$. (Here $\tilde{\rho}$ is the distance on $\bar{M}$.)

Proof. For each $x \in M$ and each $\tilde{x} \in \bar{M}$ where $\pi(\tilde{x})=x$, let $c_{x}$ be the minimizing geodesic from $x$ to $f(x)$, and $c_{\tilde{x}}$ the lift of $c_{x}$ to $\bar{M}$ starting at $\tilde{x}$. Then define $\tilde{f}(\tilde{x})=$ endpoint of $\tilde{c}_{\tilde{x}}$ over $f(x)$. $f$ obviously covers $f$ so it is an isometry of $M$. Moreover, $\tilde{c}_{x}$ is a geodesic which minimizes the distance from $\tilde{x}$ to $\tilde{f}(\tilde{x})$ and has the same length as $c_{x}$, so $\rho(x, f(x))=\tilde{\rho}(\tilde{x}, \tilde{f}(\tilde{x}))$.

Now if $\Gamma$ is the group of deck transformations of $\bar{M} \stackrel{\pi}{\longrightarrow} M$, it is evident that $\Gamma$ preserves Crit $(\bar{f})$, so that $\operatorname{Crit}(\bar{f})=\operatorname{Crit}(\bar{f}) / \Gamma$.

(2.7.3) Corollary. Let $M$ be a connected Riemannian symmetric space, and $g$ an isometry whose lifting $\bar{g}$ satisfies the hypotheses of Theorem 2.7.1. If $x \in \mathrm{Crit}(\mathrm{g})$, then the comyonent of Crit $(g)$ containing $x$ is $Z_{\tilde{G}}^{0}(g) \cdot \tilde{x} / \Gamma$, where $\pi(\tilde{x})=x$, and $\tilde{G}$ is the isometry group of $\bar{M}$.

\section{Bibliography}

[1] R. Bott, Non-degenerate critical manifolds, Ann. of Math. (2) 60 (1954) 248-261.

[2] - Vector fields and characteristic numbers, Mich. Math. J. 14 (1967) 231-244.

[ 3 ] C. Chevalley, Theory of Lie groups, Princeton University Press, Princeton, 1946.

[4] R. Crittenden, Minimum and conjugate points in symmetric spaces, Canad. J. Math. 14 (1962) 320-328.

[ 5] T. Frankel, Critical submanifolds of the classical groups, Differential and combinatorial topology, A symposium in honor of Marston Morse, Princeton University Press, Princeton, 1965.

[6] S. Helgason, Differential geometry and symmetric spaces, Academic Press, New York, 1962.

[7] R. Hermann, C-W cell decompositions of symmetric spaces, Bull. Amer. Math. Soc. 66 (1960) 126-128. [ 8 ] $\frac{298 .}{2}$. Totally geodesic orbits of groups of isometries, Indag. Math. 24 (1962) 291-

[9] S. Kobayashi, Fixed points of isometries, Nagoya Math. J. 13 (1958) 63-68.

[10] - Vector fields and characteristic numbers, unpublished. 
[11] S. Kobayashi \& K. Nomizu, Foundations of differential geometry, Vol. I, Interscience, New York, 1963.

[12] K. Nomizu, Invariant affine connections on homogeneous spaces, Amer. J. Math. 76 (1954) 33-65.

[13] J. A. Wolf, Discrete groups, symmetric spaces and global holonomy, Amer. J. Math. 84 (1962) 527-542.

[14] - Homogeneity and bounded isometries in manifolds of negative curvature, Illinois J. Math. 8 (1964) 14-18.

[15] - Spaces of constant curvature, McGraw-Hill, New York, 1967.

University of California, Bbrkeley 